\title{
¿Los menores españoles, en su tiempo libre, prefieren dispositivos electrónicos o actividad física?
}

Do Spanish minors, in their free time, prefer electronic devices or physical activity?

Brais Boente-Antela ${ }^{1,3}$, Raquel Leirós-Rodríguez ${ }^{2,3}$ y José Luis García-Soidán ${ }^{1,3 *}$

1 Facultad de Ciencias de la Educación y del Deporte. Universidade de Vigo; Campus A Xunqueira , s/n. 36005 Pontevedra. España. E-Mail: antelab@uvigo.es

2 Facultad de Fisioterapia. Universidade de Vigo; Campus A Xunqueira, s/n. 36005 Pontevedra. España. E-Mail: rleiros@uvigo.es

3 Grupo de Investigación Hi10. Universidade de Vigo; Campus A Xunqueira, s/n. 36005 Pontevedra. España. E-Mail: jlsoidan@uvigo.es

* Autor para correspondencia: José Luis García-Soidán E-Mail: jlsoidan@uvigo.es 


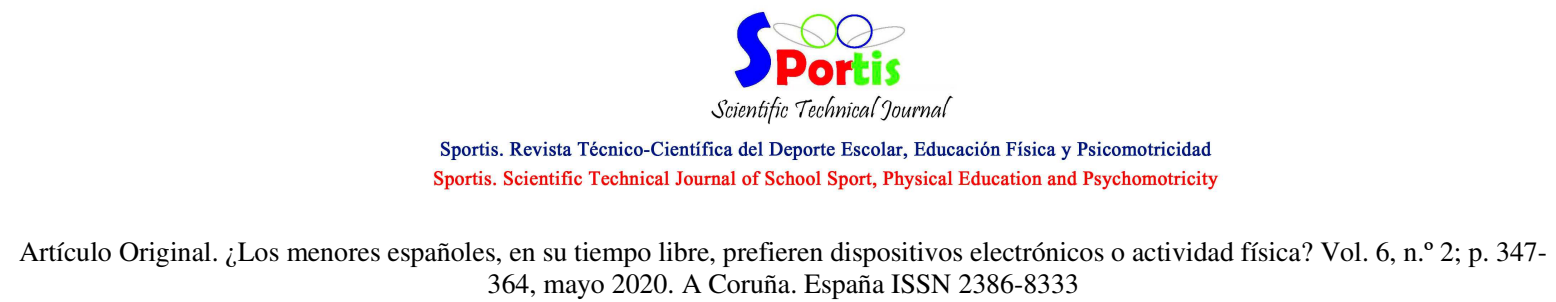

\title{
Resumen
}

Las nuevas generaciones viven expuestos diariamente a estímulos provenientes de dispositivos electrónicos y diversos instrumentos de ocio que llevan asociado su uso al mantenimiento de una conducta sedentaria. Hasta el momento no se han explorado las posibles relaciones existentes entre los diferentes componentes del estilo de vida en una muestra representativa de la población de menores españoles. Por ello, se diseñó la presente investigación con los objetivos de examinar el grado de exposición a pantallas de los niños españoles e identificar posibles relaciones en su estilo de vida entre dicho aspecto y otros hábitos como la práctica de ejercicio y las horas de descanso, así como, determinar si alguno de ellos influye en su salud mental. Los resultados obtenidos mostraron que la exposición a pantallas de los niños españoles es de 15 horas semanales, y que su estilo de vida, aunque mejorable, entra dentro de los estándares de salud en cuanto a horas de descanso y hábitos de actividad física. Las políticas preventivas de abuso de dispositivos electrónicos en menores en España deberían poner especial énfasis en el sector que peores resultados en las variables de salud mental obtuvieron en la presente investigación: niños menores de 7 años y con malos hábitos de descanso nocturno y/o más horas de exposición a pantallas.

\section{Palabras clave}

Tiempo libre; Psicología infantil; Actividad física; Estilos de vida; Videojuegos; niños.

\begin{abstract}
The new generations live exposed daily to stimuli from electronic devices that are associated with maintaining sedentary behavior. So far, the possible relationships between the different components of lifestyle have not been explored in a representative sample of the population of Spanish minors. For this reason, this research was designed with the objectives of examining the degree of exposure to screens of Spanish children and identifying possible relationships in their lifestyle and other habits such as exercising and resting hours, as well as determining whether some of them influence your mental health. The results obtained showed that the exposure of Spanish children to screens is 15 hours a week, and that their lifestyle, although it could be improved, falls within health standards in terms of hours of rest and physical activity habits. The preventive policies of abuse of electronic devices in minors in Spain should place special emphasis on the sector with the worst results in the variables of mental health obtained in the present investigation: children under 7 years of age and with poor night-time rest habits and / or more hours of exposure to screens.
\end{abstract}

\section{Key words}

Free time; Child psychology; Physical activity; Lifestyles; Video games; children.

Para citar este artículo utilice la siguiente referencia: Boente-Antela, B.; Leirós-Rodríguez, R.; García-Soidán, J.L. (2020). ¿Los menores españoles, en su tiempo libre, prefieren dispositivos electrónicos o actividad física?. Sportis Sci J, 6 (2), 347-364.

DOI: https://doi.org/10.17979/sportis.2020.6.2.6160

http://revistas.udc.es/ 


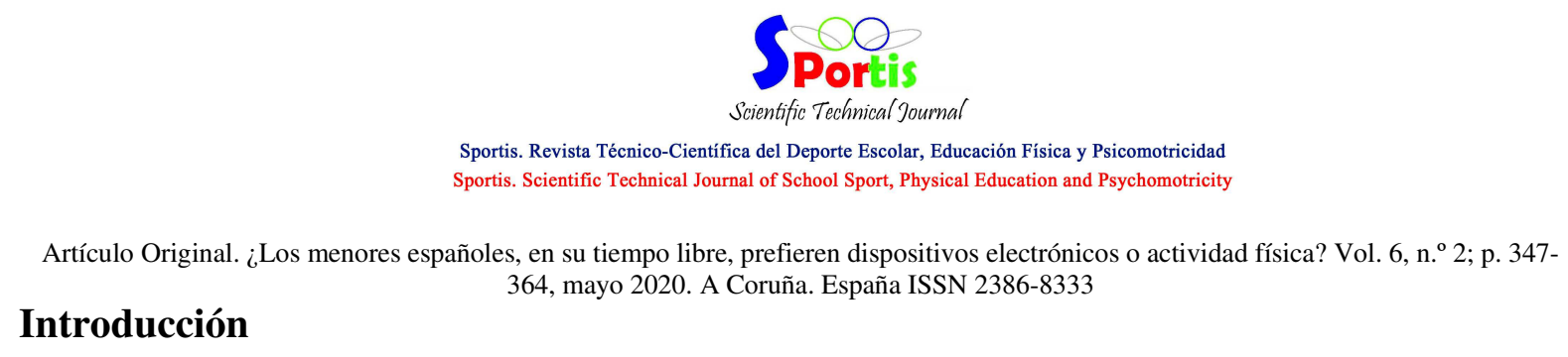

Actualmente, los dispositivos electrónicos están presentes en multitud de tareas de la vida diaria y elementos como los teléfonos móviles, tabletas, ordenadores y, por supuesto, las televisiones son herramientas de comunicación, trabajo y ocio para los adultos con los que los niños se relacionan directa e indirectamente (Brown y Smolenaers, 2018). Aunque las investigaciones realizadas hasta el momento han mostrado resultados inciertos en sus efectos para los niños, la evidencia disponible prioriza sus riesgos sobre los posibles beneficios: aumento de la incidencia de obesidad por el sedentarismo que implica el uso de dispositivos electrónicos, reducción en la capacidad de concentración y menor desarrollo de aptitudes socio-emocionales (Lissak, 2018; Livingstone y Franklin, 2018; Nightingale et al., 2017).

Paralelamente, se estima que en 2020 la prevalencia del sobrepeso infantil alcance el $20 \%$ para la población de menores escolares (De Onis, Blössner, y Borghi, 2010; Wang y Lim, 2012). Al mismo tiempo, se ha demostrado que la obesidad infantil se correlaciona con su permanencia en la etapa adulta y con mortalidad prematura (Reilly y Kelly, 2011). La práctica de actividad física (AF) conlleva una serie de reacciones fisiológicas entre las que se encuentra la segregación de neurotransmisores y hormonas que facilitan el desarrollo intelectual al facilitar la generación de nuevas neuronas y multiplicando y fortaleciendo las conexiones neuronales ya existentes entre las áreas encefálicas relacionadas con la memoria y el aprendizaje (Alfonso-Rosa, 2016; Erickson et al., 2011; Gearin y Fien, 2016). Estos fenómenos, presentes a lo largo de toda la vida, cobran especial relevancia en los niños, en los que la práctica de AF provoca mejoras en aptitudes intelectuales básicas como el control emocional, la memoria o la capacidad de adaptación a diferentes tareas y entornos que son imprescindibles para el óptimo desarrollo académico y emocional (Arufe-Giráldez, 2019; Gómez-Pinilla y Hillman, 2013; Hillman et al., 2014; Portolés-Ariño y González-Hernández, 2016).

En el contexto actual, ambos fenómenos (uso de dispositivos electrónicos y sedentarismo) están confluyendo e interaccionando entre sí, retroalimentándose uno al otro (Nightingale et al., 2017; Sánchez-Zafra, 2019). Finalmente y de manera simultánea, al escenario descrito hay que añadir el aumento de la incidencia de trastornos psicológicos

Para citar este artículo utilice la siguiente referencia: Boente-Antela, B.; Leirós-Rodríguez, R.; García-Soidán, J.L. (2020). ¿Los menores españoles, en su tiempo libre, prefieren dispositivos electrónicos o actividad física?. Sportis Sci J, 6 (2), 347-364.

DOI: https://doi.org/10.17979/sportis.2020.6.2.6160

http://revistas.udc.es/ 
emocionales como el negativista desafiante, por déficit de atención con hiperactividad o de la desregulación emocional (Monsalve-Robayo, Mora-Caro, Ramírez-López, Rozo-Hernández, y Rojas Puerto, 2017; Ruiz y Castañeda, 2016).

Sin embargo, no se han explorado las posibles relaciones exitentes entre los diferentes componentes del estilo de vida (uso de dispositivos electrónicos, hábitos de AF y horas de descanso nocturno) en muestras suficientemente amplias y representativas. Por ello, se diseñó la presente investigación con los objetivos de examinar el grado de exposición a pantallas de los menores de edad españoles e identificar posibles relaciones en su estilo de vida entre dicho aspecto y otros hábitos como la práctica de AF y las horas de descanso, así como, determinar si alguno de ellos influye en su salud mental. A su vez, se partió con las hipótesis iniciales de que los menores sí están expuestos a dispositivos electrónicos, que dicho hábito influye directamente en el tiempo invertido en la práctica de AF y, todo ello, a su vez también interviene en el estado de salud mental de los niños y niñas españoles.

\section{Material y método}

\section{Diseño experimental}

Este estudio observacional transversal se basó en los datos procedentes de la última Encuesta Nacional de Salud de España (ENS) disponible (2017) realizada por el Instituto Nacional de Estadística (INE) y el Ministerio de Sanidad, Consumo y Bienestar Social del Gobierno de España. La encuesta se realizó (mediante muestreo sistemático y con equiprobabilidad de ser seleccionados dentro de sus correspondientes secciones censales a 37500 viviendas familiares de todo el territorio nacional con entre octubre de 2016 y octubre de 2017.

\section{Muestra}

En este estudio se han empleado los datos procedentes de 3799 encuestas respondidas por un adulto que convivía con un menor de edad mayor de 5 años. Los menores de dicha edad fueron excluidos porque la cuantificación de la AF realizada por menores de esta edad sin monitorización objetiva ni observación directa carece de fiabilidad (Oliver, Schofield, y

Para citar este artículo utilice la siguiente referencia: Boente-Antela, B.; Leirós-Rodríguez, R.; García-Soidán, J.L. (2020). ¿Los menores españoles, en su tiempo libre, prefieren dispositivos electrónicos o actividad física?. Sportis Sci J, 6 (2), 347-364. DOI: https://doi.org/10.17979/sportis.2020.6.2.6160

http://revistas.udc.es/ 


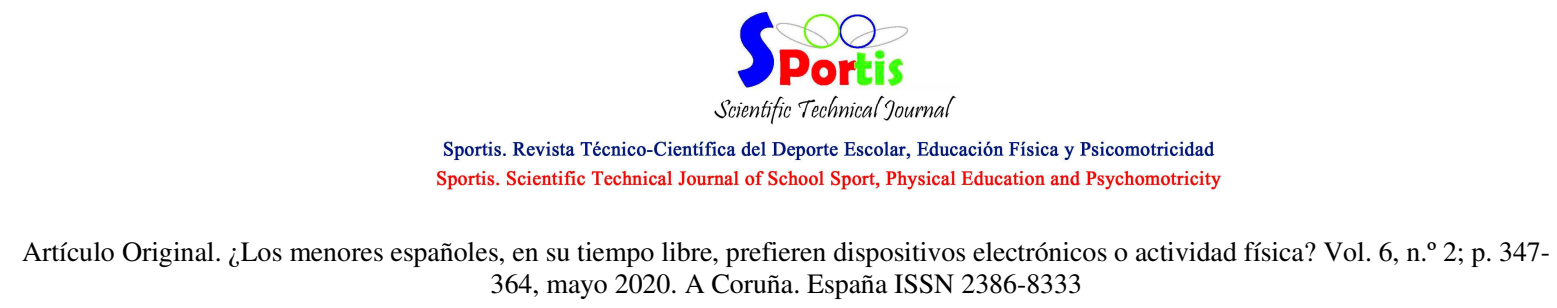

Kolt, 2007). De la muestra total la distribución por sexos fue de 50.8\% de hombres y $49.2 \%$ de mujeres y la edad media de $9.8 \pm 2.9$ años.

\section{Variables de estudio}

Como variables dependientes se consideraron:

- La frecuencia con la que el menor practica AF en el tiempo libre: a través de la pregunta ¿Cuál de estas posibilidades describe mejor la frecuencia con la que realiza alguna actividad física en su tiempo libre? Las posibilidades de respuesta fueron: (a) no hace ejercicio; (b) hace alguna actividad física o deportiva ocasional; (c) varias veces al mes); o (d) varias veces a la semana.

- La frecuencia de uso de pantallas entre semana y durante el fin de semana: estas variables en la ENS se operativizaron a través de las preguntas ¿Cuánto tiempo está frente a una pantalla (ordenador, tablet, televisión, vídeos, videojuegos, móvil...) un día entresemana? (FPLV) y ¿Cuánto tiempo está frente a una pantalla (ordenador, tablet, televisión, vídeos, videojuegos, móvil...) un día del fin de semana? (FPSD). Las posibilidades de respuesta fueron: (a) nada o casi nada; (b) menos de una hora; o (c) una hora o más.

- El número de horas diarias de uso de pantallas entre semana y en el fin de semana: operativizado a través de las preguntas ¿Cuál es el número de horas diarias que está frente a una pantalla de lunes a viernes? (HPLV) y ¿Cuál es el número de horas diarias que está frente a una pantalla sábados y domingos? (HPSD). Ambas, se registraron en escala continua numérica.

- El número de horas de descanso (HD): mediante la pregunta directa de ¿Cuántas horas duerme habitualmente al día? Se registraron el número de horas que el menor descansa habitualmente como una variable continua numérica.

- Cinco variables ofrecidas por la ENS respecto a la salud mental de los menores que evalúan la prevalencia de riesgo de mala salud mental en dicha población. Para ello, la ENS incluyó en su cuestionario el submódulo Strengths and Difficulties Questionnaire (Goodman, 2001). Con el referente temporal de los últimos seis meses, el adulto de Para citar este artículo utilice la siguiente referencia: Boente-Antela, B.; Leirós-Rodríguez, R.; García-Soidán, J.L. (2020). ¿Los menores españoles, en su tiempo libre, prefieren dispositivos electrónicos o actividad física?. Sportis Sci J, 6 (2), 347-364. 
Artículo Original. ¿Los menores españoles, en su tiempo libre, prefieren dispositivos electrónicos o actividad física? Vol. 6, n. ${ }^{\circ}$ 2; p. 347364, mayo 2020. A Coruña. España ISSN 2386-8333

referencia valoró el comportamiento que había tenido el menor en relación a: (a) síntomas emocionales; (b) problemas de conducta; (c) hiperactividad; (d) problemas con compañeros; y (e) conducta prosocial. La puntuación de estas variables puede estar entre 0 y 10 puntos, siendo 0 la mejor puntuación obtenible y 10 la peor. Sin embargo, esta escala es diferente para la puntuación correspondiente a la conducta prosocial, donde el 0 indica la peor puntuación posible y el 10 la mejor.

Las variables independientes de estudio fueron: (a) sexo; (b) edad (años); (c) peso $(\mathrm{kg})$; (d) Índice de Masa Corporal (IMC) $\left(\mathrm{kg} / \mathrm{m}^{2}\right)$, calculado a partir del peso y altura que los adultos informaban en la entrevista y clasificado en infrapeso, normopeso, sobrepeso u obesidad según las recomendaciones de la World Obesity Federation (Cole, Flegal, Nicholls, y Jackson, 2007).
\end{abstract}

\title{
Análisis estadístico
}

Para el análisis de los resultados, la muestra de menores fue dividida en tres grupos de edad: G1 de entre 5 y 7 años $(n=977), G 2$ de entre 8 y 10 años $(n=1093)$ y G3 de entre 11 y 14 años $(\mathrm{n}=1729)$.

Para caracterizar la muestra se emplearon medidas descriptivas (frecuencias, porcentajes, media, desviación típica y $95 \%$ de intervalo de confianza). El test de proporción chi-cuadrado fue empleado para comprobar la igualdad de proporciones de los grupos en una base de datos grande. Las observaciones sin valores fueron eliminadas automáticamente por el programa de análisis estadístico.

Se emplearon pruebas $\mathrm{T}$ para determinar las diferencias entre sexos y prueba ANOVA para hacerlo entre los diferentes grupos de edad. Se ha realizado, además, el análisis del coeficiente de correlación de Pearson para las variables dependientes en escala continua del estudio.

Todos los análisis estadísticos se realizaron con Stata para Mac, versión 12 y la significación estadística se estableció siempre en un valor de $\mathrm{p}<0.05$.

Para citar este artículo utilice la siguiente referencia: Boente-Antela, B.; Leirós-Rodríguez, R.; García-Soidán, J.L. (2020). ¿Los menores españoles, en su tiempo libre, prefieren dispositivos electrónicos o actividad física?. Sportis Sci J, 6 (2), 347-364.

DOI: https://doi.org/10.17979/sportis.2020.6.2.6160

http://revistas.udc.es/ 
Artículo Original. ¿Los menores españoles, en su tiempo libre, prefieren dispositivos electrónicos o actividad física? Vol. 6, n. ${ }^{\text {o }}$ 2; p. 347-

\section{Aspectos Éticos} 364, mayo 2020. A Coruña. España ISSN 2386-8333

Este estudio ha sido realizado con datos públicos anonimizados y sin posibilidad de identificar a los encuestados, procedentes de la Encuesta Nacional de Salud de España de 2017, realizada por el Ministerio de Sanidad, Consumo y Bienestar Social en colaboración con el INE. De acuerdo con la legislación española, no es necesaria la aprobación de ningún Comité Ético.

\section{Resultados}

\section{Características de la muestra}

La muestra de estudio formada por los datos de 3799 menores de edad, su distribución por sexos fue de $50.8 \%$ de hombres y $49.2 \%$ de mujeres y su edad media de $9.8 \pm 2.9$ años. Los participantes en su conjunto (independientemente del grupo de edad al que perteneciesen) presentaron normopeso (Tabla 1). Analizando por separado a ambos sexos, dentro de cada grupo de edad, no se hallaron diferencias significativas respecto a su IMC en el G1 y G3. Por el contrario, en el G2 sí se hallaron diferencias en el IMC ( $p=0.023)$, así como en el peso ( $p$ $=0.009)$. Respecto a su peso y altura, se hallaron diferencias entre sexos en el G3, $(\mathrm{p}=0.01)$ $\mathrm{y}(\mathrm{p}=0.002)$ respectivamente.

Tabla 1.

Estadística descriptiva de la muestra de estudio [datos proporcionados: media \pm desviación típica (intervalo de confianza al 95\%)]

\begin{tabular}{|c|c|c|c|c|c|c|c|}
\hline \multicolumn{8}{|c|}{ Menores $(n=3799)$} \\
\hline & \multirow[t]{2}{*}{ Todos } & \multicolumn{2}{|c|}{$\mathbf{G 1}(\mathrm{n}=977)$} & \multicolumn{2}{|c|}{ G2 $(n=1093)$} & \multicolumn{2}{|c|}{ G3 $(n=1729)$} \\
\hline & & Niños & Niñas & Niños & Niñas & Niños & Niñas \\
\hline Edad & $\begin{array}{l}9.8 \pm 2.9 \\
(9.7-9.9)\end{array}$ & $\begin{array}{l}6 \pm 0.8 \\
(6-6.1)\end{array}$ & $\begin{array}{c}6 \pm 0.8 \\
(5.9-6.1)\end{array}$ & $\begin{array}{c}9.1 \pm 0.8 \\
(9-9.2)\end{array}$ & $\begin{array}{c}9 \pm 0.8 \\
(8.9-9.1)\end{array}$ & $\begin{array}{c}12.5 \pm 1.1 \\
(12.4-12.6)\end{array}$ & $\begin{array}{c}12.5 \pm 1.1 \\
(12.4-12.6)\end{array}$ \\
\hline Peso & $\begin{array}{c}38.5 \pm 14.4 \\
(38-39)\end{array}$ & $\begin{array}{c}23.8 \pm 5.5 \\
(23.3-24.3)\end{array}$ & $\begin{array}{c}23.8 \pm 5.9 \\
(23.2-24.4)\end{array}$ & $\begin{array}{c}35.3 \pm 8.9 \\
(34.5-36.1)\end{array}$ & $\begin{array}{c}33.8 \pm 8.5^{* *} \\
(33.1-34.5)\end{array}$ & $\begin{array}{c}50.1 \pm 12.9 \\
(49.2-51)\end{array}$ & $\begin{array}{c}48.7 \pm 10.5^{*} \\
(48-49.4)\end{array}$ \\
\hline Altura & $\begin{array}{c}142 \pm 19.5 \\
(141-143)\end{array}$ & $\begin{array}{c}118.1 \pm 11.7 \\
(117-119)\end{array}$ & $\begin{array}{c}118.1 \pm 11.2 \\
(117-119)\end{array}$ & $\begin{array}{c}137.3 \pm 11.6 \\
(136-138)\end{array}$ & $\begin{array}{c}136.6 \pm 10.4 \\
(136-138)\end{array}$ & $\begin{array}{c}158.1 \pm 12.3 \\
(157-159)\end{array}$ & $\begin{array}{c}156.5 \pm 9.8 * * \\
(156-157)\end{array}$ \\
\hline IMC & $\begin{array}{c}18.8 \pm 4.1 \\
(18.7-18.9)\end{array}$ & $\begin{array}{c}17.4 \pm 5.4 \\
(16.9-17.9)\end{array}$ & $\begin{array}{c}17.2 \pm 4.4 \\
(16.8-17.6)\end{array}$ & $\begin{array}{c}18.6 \pm 3.9 \\
(18.3-18.9)\end{array}$ & $\begin{array}{l}18.1 \pm 3.5^{*} \\
(17.8-18.4)\end{array}$ & $\begin{array}{c}19.9 \pm 3.6 \\
(19.6-20.2)\end{array}$ & $\begin{array}{c}19.9 \pm 3.5 \\
(19.7-20.1)\end{array}$ \\
\hline HPLV & $\begin{array}{l}2.1 \pm 1.2 \\
(2.1-2.1)\end{array}$ & $\begin{array}{l}1.9 \pm 1 \\
(1.8-2)\end{array}$ & $\begin{array}{c}1.8 \pm 0.9^{* \mathrm{~b} \#} \\
(1.7-1.9)\end{array}$ & $\begin{array}{c}2.1 \pm 1.1(2- \\
2.2)\end{array}$ & $\begin{array}{c}1.8 \pm 0.9^{* *} \\
(1.7-1.9)\end{array}$ & $\begin{array}{l}2.3 \pm 1.2 \\
(2.2-2.4)\end{array}$ & $\begin{array}{c}2.4 \pm 1.3^{\mathrm{b \#}} \\
(2.3-2.5)\end{array}$ \\
\hline
\end{tabular}

Para citar este artículo utilice la siguiente referencia: Boente-Antela, B.; Leirós-Rodríguez, R.; García-Soidán, J.L. (2020). ¿Los menores españoles, en su tiempo libre, prefieren dispositivos electrónicos o actividad física?. Sportis Sci J, 6 (2), 347-364. DOI: https://doi.org/10.17979/sportis.2020.6.2.6160 
Artículo Original. ¿Los menores españoles, en su tiempo libre, prefieren dispositivos electrónicos o actividad física? Vol. 6, n. ${ }^{\text {o }}$ 2; p. 347364, mayo 2020. A Coruña. España ISSN 2386-8333

\begin{tabular}{|c|c|c|c|c|c|c|c|}
\hline HPS & $\begin{array}{c}2.9 \pm 1.4 \\
(2.9-3)\end{array}$ & $\begin{array}{l}2.6 \pm 1.2 \\
(2.5-2.7)\end{array}$ & $\begin{array}{c}2.4 \pm \\
1.1 * * \mathrm{a} \mathrm{a} \mathrm{b} \# \\
(2.3-2.5)\end{array}$ & $\begin{array}{c}2.9 \pm 1.4 \\
(2.8-3)\end{array}$ & $\begin{array}{c}2.6 \pm \\
1.2 * * \mathrm{a \# c \#} \\
(2.5-2.7)\end{array}$ & $\begin{array}{c}3.1 \pm 1.4 \\
(3-3.2)\end{array}$ & $\begin{array}{c}3.2 \pm 1.5^{\mathrm{b} \# \mathrm{c} \#} \\
(3.1-3.3)\end{array}$ \\
\hline HD & $\begin{array}{c}9.2 \pm 1 \\
(9.2-9.2)\end{array}$ & $\begin{array}{c}9.7 \pm 1 \\
(9.6-9.8)\end{array}$ & $\begin{array}{c}9.7 \pm 1^{\text {a\#b\# }} \\
(9.6-9.8)\end{array}$ & $\begin{array}{l}9.4 \pm 0.9 \\
(9.3-9.5)\end{array}$ & $\begin{array}{c}9.4 \pm 1^{\text {a\#c\# }} \\
(9.3-9.5)\end{array}$ & $\begin{array}{l}8.8 \pm 0.9 \\
(8.7-8.9)\end{array}$ & $\begin{array}{c}8.8 \pm 1^{\mathrm{b \# c} \#} \\
(8.7-8.9)\end{array}$ \\
\hline
\end{tabular}

G1: entre 5 y 7 años; G2: entre de 8 y 10 años; G3: entre 11 y 14 años.

Edad (años); Peso (kg); Altura (cm); IMC: índice de masa corporal $\left(\mathrm{kg} / \mathrm{m}^{2}\right)$; HPLV: horas frente a una pantalla entre semana (horas); HPSD: horas frente a una pantalla en fin de semana (horas); HD: horas de descanso nocturno (horas).

$\mathrm{a}=\mathrm{G} 1$ vs. G2; $\mathrm{b}=\mathrm{G} 1$ vs. G3; $\mathrm{c}=\mathrm{G} 2$ vs. G3.

$* \mathrm{p}<0.05, \mathrm{t}$-test; $* * \mathrm{p}<0.01, \mathrm{t}$-test; $\# \mathrm{p}<0.001$, ANOVA

En lo concerniente a las horas de exposición a pantallas (Tabla 1), se identificaron diferencias para: HPLV entre G1 y G3 ( $\mathrm{p}<0.001$ ); y para HPSD y HD entre todos los grupos ( $p<0.001$, para ambas variables). Respecto al uso de pantallas, el G3 es el que mayor uso presentó tanto entre semana como los fines de semana. Inversamente, en las horas de descanso se pudo apreciar un descenso a medida que se avanzaba la edad ( $p<0.0001)$. De entre dichas variables, solamente se correlacionaron HPLV y HPSD $(r=0.6 ; p=0.0001)$.

\section{Relación entre la edad y frecuencia de uso de pantallas}

Los datos de FPLV reflejan que los niños, en conjunto, hacen mayor uso de los dispositivos electrónicos que las niñas (Tabla 2). Sin embargo, a medida que se incrementaba la edad, la exposición a pantallas también lo hizo (sobre todo en las niñas).

Tabla 2.

Estadística descriptiva de la frecuencia de uso de pantallas [datos proporcionados: $n$ (porcentaje)]

\begin{tabular}{lcccccc|ccc}
\hline & \multicolumn{2}{c}{ G1 $(\mathrm{n}=976)$} & \multicolumn{2}{c|}{ G2 $(\mathrm{n}=1092)$} & \multicolumn{2}{c}{$\mathbf{G 3}(\mathrm{n}=1725)$} & \multicolumn{2}{c}{ Total } \\
$(\mathrm{n}=3793)$
\end{tabular}

Para citar este artículo utilice la siguiente referencia: Boente-Antela, B.; Leirós-Rodríguez, R.; García-Soidán, J.L. (2020). ¿Los menores españoles, en su tiempo libre, prefieren dispositivos electrónicos o actividad física?. Sportis Sci J, 6 (2), 347-364. 
Artículo Original. ¿Los menores españoles, en su tiempo libre, prefieren dispositivos electrónicos o actividad física? Vol. 6, n. ${ }^{\circ}$ 2; p. 347 -

\begin{tabular}{lcccccc|cc} 
& \multicolumn{8}{c}{364 , mayo 2020. A Coruña. España ISSN 2386-8333 } \\
Menos de na & 47 & 36 & 43 & 39 & 42 & 38 & 132 & 113 \\
hora & $(35.6)$ & $(31.9)$ & $(32.6)$ & $(34.5)$ & $(31.8)$ & $(33.6)$ & $(53.9)$ & $(46.1)$ \\
Una hora o & 458 & 389 & 504 & 470 & 771 & 824 & 1733 & 1683 \\
más & $(26.4)$ & $(23.1)$ & $(29.1)$ & $(27.9)$ & $(44.5)$ & $(49)$ & $(50.7)$ & $(49.3)$ \\
& 524 & 450 & 569 & 523 & 832 & 893 & $1925^{*}$ & $1866^{*}$ \\
Total & $(27.2)$ & $(24.1)$ & $(29.6)$ & $(28)$ & $(43.2)$ & $(47.9)$ & $(50.8)$ & $(49.2)$ \\
\end{tabular}

G1: entre 5 y 7 años; G2: entre de 8 y 10 años; G3: entre 11 y 14 años.

FPLV: frecuencia en el uso de pantallas de entre semana; FPSD: frecuencia en el uso de pantallas el fin de semana.

$* \mathrm{p}<0.05 ; * * \mathrm{p}<0.01$

Tras la prueba chi-cuadrado, se asumió la hipótesis alternativa de dependencia entre grupo de edad y FPLV $\left(x^{2}=45.5 ; \mathrm{p}<0.001\right)$ para la muestra en conjunto; para los niños $\left(x^{2}=\right.$ $23 ; \mathrm{p}<0.001) ; \mathrm{y}$, para las niñas $\left(x^{2}=24.3 ; \mathrm{p}<0.001\right)$.

Para la variable FPSD, la prueba chi-cuadrado solamente tuvo resultados significativos para el total de la muestra $\left(x^{2}=24.2 ; \mathrm{p}<0.001\right)$. La FPLV y FPSD no se correlacionaron las HD en ningún subgrupo muestral ni para la muestra en total.

\section{Relación entre la edad y las puntuaciones de salud mental}

Tabla 3.

Estadística descriptiva de las puntuaciones de variables de salud mental de la muestra de estudio [datos proporcionados: media \pm desviación típica (intervalo de confianza al 95\%)]

\begin{tabular}{|c|c|c|c|c|c|c|c|}
\hline & \multicolumn{2}{|c|}{$\mathbf{G 1}(\mathrm{n}=974)$} & \multicolumn{2}{|c|}{$\mathbf{G 2}(\mathrm{n}=1090)$} & \multicolumn{2}{|c|}{$\mathbf{G 3}(\mathrm{n}=1720)$} & \multirow[t]{2}{*}{ Total } \\
\hline & Niños & Niñas & Niños & Niñas & Niños & Niñas & \\
\hline SE & $\begin{array}{l}1.7 \pm 1.9 \\
(1.5-1.9)\end{array}$ & $\begin{array}{c}1.4 \pm 1.6 * * \\
(1.3-1.6)\end{array}$ & $\begin{array}{l}1.5 \pm 1.7 \\
(1.4-1.6)\end{array}$ & $\begin{array}{l}1.6 \pm 1.8 \\
(1.5-1.8)\end{array}$ & $\begin{array}{l}1.6 \pm 1.8 \\
(1.5-1.7)\end{array}$ & $\begin{array}{l}1.6 \pm 1.8 \\
(1.5-1.7)\end{array}$ & $\begin{array}{l}1.6 \pm 1.8 \\
(1.5-1.7)\end{array}$ \\
\hline $\mathrm{PC}$ & $\begin{array}{l}1.7 \pm 1.6 \\
(1.6-1.8)\end{array}$ & 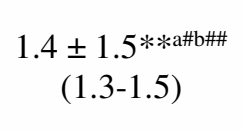 & $\begin{array}{l}1.5 \pm 1.5 \\
(1.4-1.6)\end{array}$ & $\begin{array}{c}1.2 \pm \\
1.5 * * \text { a\# } \\
(1.1-1.3)\end{array}$ & $\begin{array}{l}1.4 \pm 1.5 \\
(1.3-1.5)\end{array}$ & $\begin{array}{c}1.3 \pm 1.5^{\mathrm{b} \#} \\
(1.2-1.4)\end{array}$ & $\begin{array}{l}1.4 \pm 1.5 \\
(1.4-1.5)\end{array}$ \\
\hline $\mathrm{HA}$ & $\begin{array}{l}4.4 \pm 2.6 \\
(4.2-4.6)\end{array}$ & 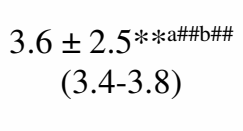 & $\begin{array}{l}3.9 \pm 2.6 \\
(3.7-4.1)\end{array}$ & 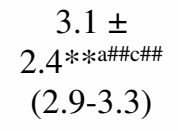 & $\begin{array}{l}3.5 \pm 2.6 \\
(3.3-3.7)\end{array}$ & $\begin{array}{c}2.8 \pm 2.4 * * \mathrm{~b} \# \text { \#c\#\# } \\
(2.6-3)\end{array}$ & $\begin{array}{l}3.5 \pm 2.6 \\
(3.4-3.6)\end{array}$ \\
\hline $\mathrm{PC}$ & $\begin{array}{c}1.1 \pm 1.5 \\
(1-1.2)\end{array}$ & $\begin{array}{c}1 \pm 1.2 \\
(0.9-1.1)\end{array}$ & $\begin{array}{c}1 \pm 1.3 \\
(0.9-1.1)\end{array}$ & $\begin{array}{c}0.9 \pm 1.3 \\
(0.8-1)\end{array}$ & $\begin{array}{c}1.1 \pm 1.5 \\
(1-1.2)\end{array}$ & $\begin{array}{c}1.1 \pm 1.4 \\
(1-1.2)\end{array}$ & $\begin{array}{l}1.1 \pm 1.4 \\
(1.1-1.1)\end{array}$ \\
\hline $\mathrm{CP}$ & $\begin{array}{l}8.8 \pm 1.5 \\
(8.7-8.9)\end{array}$ & $\begin{array}{c}9.2 \pm 1.4^{* *} \\
(9.1-9.3)\end{array}$ & $\begin{array}{c}9 \pm 1.4 \\
(8.9-9.1)\end{array}$ & $\begin{array}{c}9.3 \pm 1.2^{* *} \\
(9.2-9.4)\end{array}$ & $\begin{array}{c}9 \pm 1.4 \\
(8.9-9.1)\end{array}$ & $\begin{array}{c}9.2 \pm 1.4^{*} \\
(9.1-9.3)\end{array}$ & $\begin{array}{c}9.1 \pm 1.4 \\
(9-9.1)\end{array}$ \\
\hline
\end{tabular}

G1: entre 5 y 7 años; G2: entre de 8 y 10 años; G3: entre 11 y 14 años. 


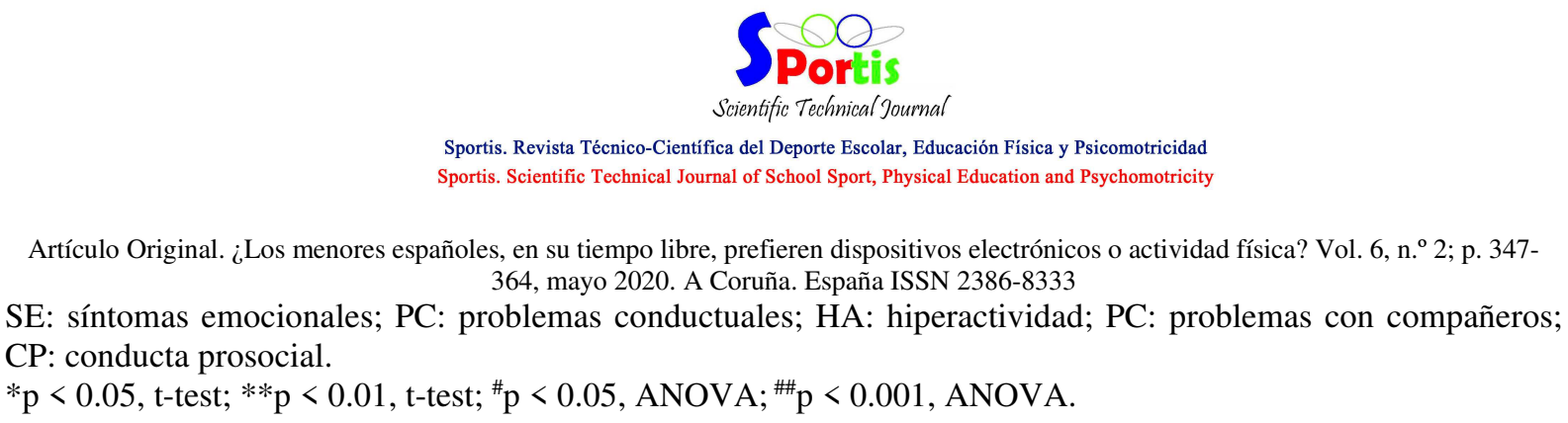

En la variable Sintomas emocionales solamente se hallaron diferencias significativas entre ambos sexos en el G1 ( $\mathrm{p}=0.002)$, siendo los niños los que peor puntuación obtuvieron (Tabla 3).

La puntuación relacionada con Problemas de conducta fue, junto con la de Hiperactividad, la que más diferencias arrojó entre subgrupos de edad y sexo. En Problemas de conducta se encontraron diferencias entre ambos sexos en G1 ( $\mathrm{p}=0.005)$ y G2 ( $\mathrm{p}=$ 0.008), como consecuencia de las peores puntuaciones de los niños en todos los subgrupos de edad. El ítem Hiperactividad fue la peor puntuación de entre las cinco variables de salud mental en ambos sexos. En este apartado, los niños también obtuvieron peores puntuaciones con diferencias significativas en todos los subgrupos de edad ( $p<0.001)$, siendo el G1 el que peores puntuaciones obtuvo y las niñas del G3 las que mejor (2.8 puntos sobre 10) (Tabla 3).

La variable Conducta prosocial fue la que presentó los mejores resultados. Las diferencias entre sexos fueron significativas en todos los subgrupos ( $p<0.0002$ en G1 y G2 y $\mathrm{p}=0.03$ en el G3). Las niñas del G2 y del G3 fueron las que presentaron las mejores puntuaciones, con un 9.3 y 9.2 sobre 10 , respectivamente.

En relación al ítem Problemas con compañeros, fue la única que no mostró diferencias entre sexos dentro de cada subgrupo de edad ni para el total de la muestra (Tabla 3).

En el análisis de correlación, las variables HPLV, HPSD y HD se asociaron significativamente con todas las variables de salud mental $(0.05<\mathrm{r}>0.1 ; \mathrm{p}<0.0001)$, exceptuando Hiperactividad que no se asoció con HD.

\section{Relación entre la frecuencia de AF y el uso de pantallas}

La primera tendencia que se pudo observar es la del aumento en la frecuencia de la exposición a pantallas a medida que también lo hace la frecuencia de AF, siendo el porcentaje de niños que no realizan AF y usan pantallas más de una hora diaria entre semana de un

Para citar este artículo utilice la siguiente referencia: Boente-Antela, B.; Leirós-Rodríguez, R.; García-Soidán, J.L. (2020). ¿Los menores españoles, en su tiempo libre, prefieren dispositivos electrónicos o actividad física?. Sportis Sci J, 6 (2), 347-364.

DOI: https://doi.org/10.17979/sportis.2020.6.2.6160

http://revistas.udc.es/ 
Artículo Original. ¿Los menores españoles, en su tiempo libre, prefieren dispositivos electrónicos o actividad física? Vol. 6, n. ${ }^{\text {o }}$ 2; p. 347364, mayo 2020. A Coruña. España ISSN 2386-8333

$10.5 \%$, frente a un $38.1 \%$ de niños que realizan AF varias veces a la semana (Tabla 4). En niñas, la diferencia no fue tan acusada, siendo de entre todas las que usan más de una hora diaria entre semana las pantallas un $18.7 \%$ las que no realizan AF y un $29.2 \%$ las que la practican varias veces a la semana.

Tabla 4.

Distribución de la frecuencia de actividad física en función de la frecuencia de uso de pantallas [datos proporcionados: $n$ (porcentaje)]

\begin{tabular}{|c|c|c|c|c|c|c|c|c|c|}
\hline \multicolumn{10}{|c|}{ Frecuencia de Actividad Física } \\
\hline \multirow[t]{2}{*}{ FPLV } & \multicolumn{2}{|c|}{ Nada } & \multicolumn{2}{|c|}{ Ocasionalmente } & \multicolumn{2}{|c|}{ Mensualmente } & \multicolumn{2}{|c|}{ Semanalmente } & \multirow[t]{2}{*}{ Total } \\
\hline & Niños & Niñas & Niños & Niñas & Niños & Niñas & Niños & Niñas & \\
\hline Nada o casi nada & $\begin{array}{c}14 \\
(15.7)\end{array}$ & $\begin{array}{c}17 \\
(20)\end{array}$ & $\begin{array}{c}10 \\
(11.2)\end{array}$ & $\begin{array}{c}16 \\
(18.8)\end{array}$ & $\begin{array}{c}29 \\
(32.6)\end{array}$ & $\begin{array}{c}29 \\
(34.1)\end{array}$ & $\begin{array}{c}36 \\
(40.4)\end{array}$ & $\begin{array}{c}23 \\
(27.1)\end{array}$ & $\begin{array}{c}174 \\
(100)\end{array}$ \\
\hline $\begin{array}{l}\text { Menos de una } \\
\text { hora }\end{array}$ & $\begin{array}{c}26 \\
(9.5)\end{array}$ & $\begin{array}{c}39 \\
(13.4)\end{array}$ & $\begin{array}{c}33 \\
(12)\end{array}$ & $\begin{array}{c}73 \\
(25.1)\end{array}$ & $\begin{array}{c}103 \\
(37.6)\end{array}$ & $\begin{array}{c}88 \\
(30.2)\end{array}$ & $\begin{array}{c}112 \\
(40.9)\end{array}$ & $\begin{array}{c}91 \\
(31.3)\end{array}$ & $\begin{array}{l}565 \\
(100)\end{array}$ \\
\hline Una hora o más & $\begin{array}{c}164 \\
(10.5)\end{array}$ & $\begin{array}{c}279 \\
(18.7)\end{array}$ & $\begin{array}{c}304 \\
(19.4)\end{array}$ & $\begin{array}{c}369 \\
(24.7)\end{array}$ & $\begin{array}{l}500 \\
(32)\end{array}$ & $\begin{array}{c}407 \\
(27.3)\end{array}$ & $\begin{array}{c}595 \\
(38.1)\end{array}$ & $\begin{array}{c}436 \\
(29.2)\end{array}$ & $\begin{array}{l}3054 \\
(100)\end{array}$ \\
\hline Total & $\begin{array}{r}204 \\
(5.4) \\
\end{array}$ & $\begin{array}{r}335 \\
(8.8) \\
\end{array}$ & $\begin{array}{r}347 \\
(9.1) \\
\end{array}$ & $\begin{array}{c}458 \\
(12.1) \\
\end{array}$ & $\begin{array}{r}632 \\
(16.6) \\
\end{array}$ & $\begin{array}{c}524 \\
(13.8) \\
\end{array}$ & $\begin{array}{c}743 \\
(19.6) \\
\end{array}$ & $\begin{array}{c}550 \\
(14.5) \\
\end{array}$ & $\begin{array}{l}3793 * \\
(100)\end{array}$ \\
\hline FPSD & & & Ocasio & lmente & Mens & mente & Seman & mente & Total \\
\hline Nada o casi nada & $\begin{array}{c}8 \\
(13.3)\end{array}$ & $\begin{array}{l}14 \\
(20)\end{array}$ & $\begin{array}{c}23 \\
(38.3)\end{array}$ & $\begin{array}{c}22 \\
(31.4)\end{array}$ & $\begin{array}{c}13 \\
(21.7)\end{array}$ & $\begin{array}{c}20 \\
(28.6)\end{array}$ & $\begin{array}{c}16 \\
(26.7)\end{array}$ & $\begin{array}{c}14 \\
(20)\end{array}$ & $\begin{array}{l}130 \\
(100)\end{array}$ \\
\hline $\begin{array}{l}\text { Menos de una } \\
\text { hora }\end{array}$ & $\begin{array}{c}17 \\
(12.9)\end{array}$ & $\begin{array}{c}29 \\
(25.7)\end{array}$ & $\begin{array}{c}19 \\
(14.4)\end{array}$ & $\begin{array}{c}28 \\
(24.8)\end{array}$ & $\begin{array}{c}59 \\
(44.7)\end{array}$ & $\begin{array}{c}30 \\
(26.5)\end{array}$ & $\begin{array}{c}37 \\
(28)\end{array}$ & $\begin{array}{c}26 \\
(23)\end{array}$ & $\begin{array}{l}245 \\
(100)\end{array}$ \\
\hline Una hora o más & $\begin{array}{c}178 \\
(10.3)\end{array}$ & $\begin{array}{c}292 \\
(17.3)\end{array}$ & $\begin{array}{c}305 \\
(17.6)\end{array}$ & $\begin{array}{c}409 \\
(24.3)\end{array}$ & $\begin{array}{c}560 \\
(32.3)\end{array}$ & $\begin{array}{c}474 \\
(28.2)\end{array}$ & $\begin{array}{c}690 \\
(39.8)\end{array}$ & $\begin{array}{c}508 \\
(30.2)\end{array}$ & $\begin{array}{l}3416 \\
(100)\end{array}$ \\
\hline Total & $\begin{array}{r}204 \\
(5.4) \\
\end{array}$ & $\begin{array}{c}335 \\
(8.8) \\
\end{array}$ & $\begin{array}{r}347 \\
(9.2) \\
\end{array}$ & $\begin{array}{c}458 \\
(12.1) \\
\end{array}$ & $\begin{array}{c}632 \\
(16.7) \\
\end{array}$ & $\begin{array}{c}524 \\
(13.8) \\
\end{array}$ & $\begin{array}{c}743 \\
(19.6) \\
\end{array}$ & $\begin{array}{c}550 \\
(14.5) \\
\end{array}$ & $\begin{array}{c}3791 * * \\
(100)\end{array}$ \\
\hline
\end{tabular}

FPLV: frecuencia en el uso de pantallas de entre semana; FPSD: frecuencia en el uso de pantallas el fin de semana.

$* \mathrm{p}<0.05 ; * * \mathrm{p}<0.01$

El resultado de la prueba chi-cuadrado de independencia entre ambas variables fue de $x^{2}=14.8(\mathrm{p}=0.02)$ para los niños, de $x^{2}=7.7(\mathrm{p}=0.02)$ para las niñas, $\mathrm{y}$ de $x^{2}=14.3(\mathrm{p}=$ 0.02) para el total de la muestra. En las variables FPLV y FPSD se encontraron diferencias en el comportamiento a lo largo de la semana solamente en aquellos niños y niñas que realizan AF varias veces a la semana y menos usan las pantallas que, durante el fin de semana aumentan el tiempo expuestos a las mismas ( $\mathrm{p}<0.0001)$ (Tabla 4).

\section{Discusión}

Para citar este artículo utilice la siguiente referencia: Boente-Antela, B.; Leirós-Rodríguez, R.; García-Soidán, J.L. (2020). ¿Los menores españoles, en su tiempo libre, prefieren dispositivos electrónicos o actividad física?. Sportis Sci J, 6 (2), 347-364. DOI: https://doi.org/10.17979/sportis.2020.6.2.6160 


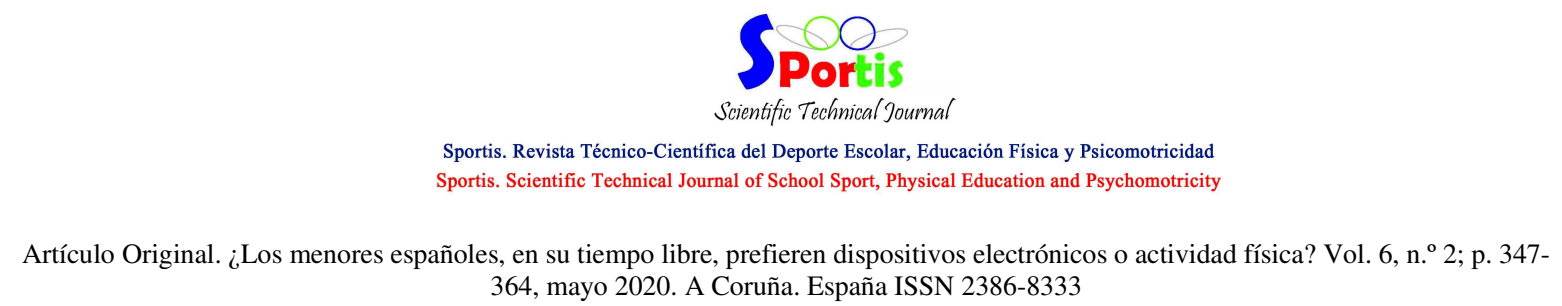

Los objetivos de la presente investigación fueron examinar el grado de exposición a pantallas de los menores de edad españoles e identificar posibles relaciones en su estilo de vida entre dicho aspecto y otros hábitos como la práctica de AF y las horas de descanso, así como, determinar si alguno de ellos influye en su salud mental. A la luz de los resultados obtenidos, en la población de niños y niñas españoles no se detectó que hubiera una asociación entre las HPLV y HPSD con las HD pero sí con peores resultados en variables relacionadas con la salud mentar y los hábitos de AF.

Concretamente, en relación a la no asociación entre las HPLV ni HPSD con las HD, aunque contradictoria con los consejos generales de evitar el uso de pantallas para facilitar el descanso de los menores (Abalde-Amoedo, y Pino-Juste, 2015; Twenge, Hisler, y Krizan, 2019); hay que señalar que dicha recomendación se ha establecido en base a menores que tenían pantallas en su habitación (es decir, con acceso totalmente libre e individual a las mismas) y, especialmente, a dispositivos portátiles (teléfonos móviles y tabletas) (Duggan, Taveras, Gerber, Horan, y Oreskovic, 2019), diferenciación que no se realiza en la ENS española. Al mismo tiempo, señalar que las asociaciones realizadas entre uso de pantallas digitales y menor horas de descanso nocturno se ha hecho con el estudio de poblaciones de menor tamaño y que reportaban un uso promedio de dispositivos con pantalla de más de 30 horas semanales (Emond y Benjamin-Neelon, 2019) (frente al promedio de 15 horas semanales de los niños españoles).

En el análisis diferenciado por sexos, los niños mostraron una mayor exposición a pantallas en todas las franjas de edad estudiadas. Aunque la diferencia entre sexos se fue reduciendo conforme avanzó la edad los participantes. Esto concuerda con investigaciones anteriores que también identificaron peores hábitos en el estilo de vida en los niños en comparación con las niñas (Keane, Kelly, Molcho, y Gabhainn, 2017). Y, del mismo modo que en los resultados obtenidos por Keane et al. (2017), los niños también mostraron peores resultados en las variables de salud mental que las niñas. Concretamente, los niños son más sensibles al desarrollo de problemas conductuales y de hiperactividad, sobre todo cuando son más pequeños (menores de siete años).

Para citar este artículo utilice la siguiente referencia: Boente-Antela, B.; Leirós-Rodríguez, R.; García-Soidán, J.L. (2020). ¿Los menores españoles, en su tiempo libre, prefieren dispositivos electrónicos o actividad física?. Sportis Sci J, 6 (2), 347-364. DOI: https://doi.org/10.17979/sportis.2020.6.2.6160

http://revistas.udc.es/ 


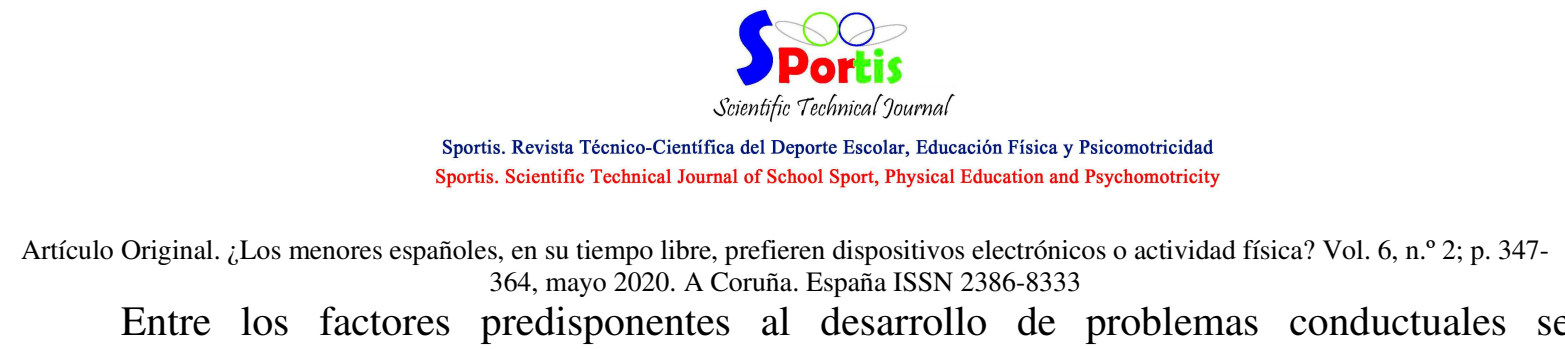
encontraron las HD y horas de exposición a pantallas, en concordancia con investigaciones previas (Castro-Sánchez, Linares-Manrique, Sanromán-Mata, y Pérez Cortés, 2017; Sezen et al., 2016; Tandon et al., 2019). Sin embargo, no se halló relación entre alteración de la salud mental y menor práctica de AF, otra asociación comúnmente hallada en la literatura existente ya mencionada. Probablemente, en este caso, como los niños que más practicaban ejercicio también eran los que más horas pasaban frente a una pantalla, este estudio ha servido para discernir cuál de las dos variables tiene realmente un efecto directo sobre el desarrollo de problemas conductuales.

En relación a los hábitos de AF fue donde se encontraron los resultados más controvertidos ya que a mayor práctica de ejercicio, más frecuencia de uso de pantallas en general y, sobre todo, durante el fin de semana. Dicha asociación, especialmente fuerte en el subgrupo de niños, no atiende a justificaciones teóricas previamente establecidas si no que puede tener explicación considerando que el uso de dispositivos con pantalla puede emplearse en el hogar como recompensa positiva o de refuerzo ante el cumplimiento de conductas positivas como realizar AF. Señalar que, aunque la mayoría de publicaciones disponibles hasta el momento apoyan la relación inversa entre tiempo dedicado a la práctica de AF y tiempo de exposición a pantallas, Maher et al. (2019), recientemente, tampoco encontró dicha relación en un estudio que incluyó a más de 1000 niños.

Por tanto, tras todo lo expuesto anteriormente, se debe tener en cuenta que: (a) la exposición a pantallas de los niños españoles es mucho menor que la detectada en otras poblaciones (Brown y Smolenaers, 2018; Duggan et al., 2019; Emond y Benjamin-Neelon, 2019; Hale y Guan, 2015); (b) que este fenómeno concuerda con la identificación de su peso como normal (sin prevalencia evidente de sobrepeso y obesidad ante la falta de un elevado número de horas en sedestación frente a dispositivos electrónicos); y (c) que su estilo de vida, aunque mejorable, entra dentro de los estándares de salud en cuanto a HD y hábitos de AF (World Health Organization, 2019).

Para citar este artículo utilice la siguiente referencia: Boente-Antela, B.; Leirós-Rodríguez, R.; García-Soidán, J.L. (2020). ¿Los menores españoles, en su tiempo libre, prefieren dispositivos electrónicos o actividad física?. Sportis Sci J, 6 (2), 347-364. DOI: https://doi.org/10.17979/sportis.2020.6.2.6160

http://revistas.udc.es/ 


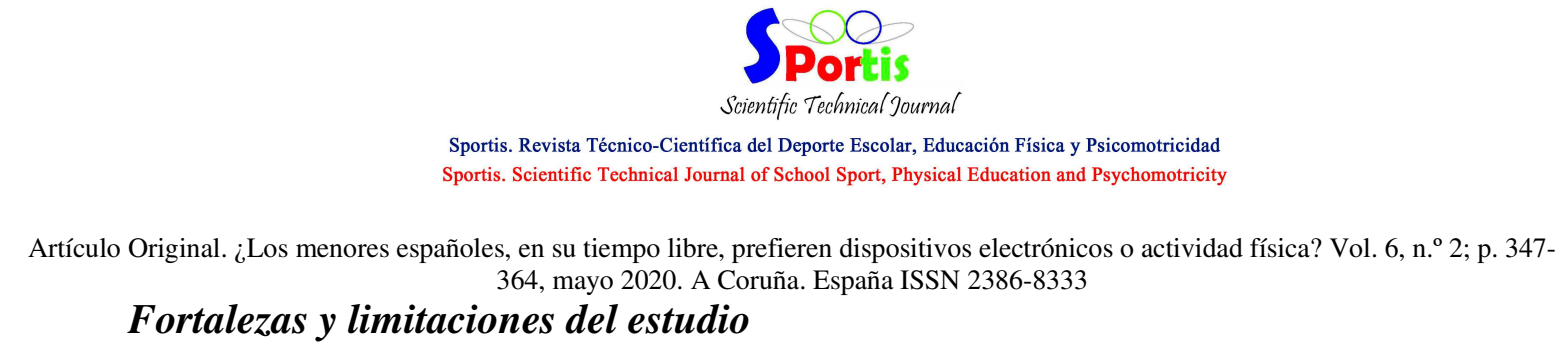

Este estudio tiene importantes limitaciones que deben ser reconocidas. Como principal factor limitante, los resultados, y las encuestas de los que se obtuvieron, no han sido obtenidos por los investigadores de este estudio, ya que son procedentes del trabajo colaborativo entre el INE y el Ministerio de Sanidad, Consumo y Bienestar Social de España. Primero, se ha empleado información auto-reportada sobre las variables de estudio en lugar de mediciones realizadas por un evaluador experto. En segundo lugar, la población exclusivamente española limita la generalización de nuestros resultados a otras poblaciones. En tercer lugar, él hábito de AF también fue autorreportado y calificado por el participante en términos de frecuencia sin haberse cuantificado objetivamente mediante podómetro o acelerómetro en términos de cantidad, frecuencia e intensidad de la AF realizada.

A pesar de las limitaciones antes mencionadas, también hay fortalezas importantes. Se trata de un estudio poblacional amplio que, aunque con cautela, es representativa de la población española y reflejo real la situación actual de los hábitos de exposición a pantallas y de práctica de AF de las nuevas generaciones. Al mismo tiempo, es la primera vez que se detectan relaciones directas entre el estilo de vida de los menores y el desarrollo de alteraciones en la salud mental, habiendo variables y asociaciones que se comportan de manera similar a la de otras poblaciones, pero, en otros casos, habiendo particularidades para la población española.

\section{Conclusiones}

Independientemente de que el uso y abuso de pantallas durante la infancia es una conducta dependiente de múltiples factores como la edad, el sexo y el estilo de vida de los padres. Su empleo durante la edad escolar, hasta que no se confirme que es inocuo para el correcto desarrollo psicomotriz de los niños, debiera restringirse dando prioridad al empleo del tiempo libre en la práctica de AF (cuyos beneficios en el desarrollo sí están plenamente contrastados).

Además, deben aplicarse políticas de Salud Pública con la mayor brevedad posible para reducir la incidencia de alteraciones en la salud mental de los menores. Teniendo dichas

Para citar este artículo utilice la siguiente referencia: Boente-Antela, B.; Leirós-Rodríguez, R.; García-Soidán, J.L. (2020). ¿Los menores españoles, en su tiempo libre, prefieren dispositivos electrónicos o actividad física?. Sportis Sci J, 6 (2), 347-364.

DOI: https://doi.org/10.17979/sportis.2020.6.2.6160

http://revistas.udc.es/ 
intervenciones como población diana, especialmente, a los niños menores de 7 años y con malos hábitos de descanso nocturno y/o más horas de exposición a pantallas.

\section{Referencias bibliográficas}

Abalde-Amoedo, N., y Pino-Juste, M. (2015). Influencia de la actividad física y el sobrepeso en el rendimiento académico: revisión teórica. Sportis. Scientific Journal of School Sport, Physical Education and Psychomotricity, 2(1), 147-161. DOI: https://doi.org/10.17979/sportis.2016.2.1.1446

Alfonso-Rosa, R. (2016). Relación entre la actividad física extraescolar y el rendimiento académico en alumnos de Educación Secundaria. Sportis. Scientific Journal of School Sport, Physical Education and Psychomotricity, 2(2), 177-187. DOI: https://doi.org/10.17979/sportis.2016.2.2.1428

Arufe Giráldez, Víctor (2019). Fortnite EF un nuevo juego deportivo para el aula de Educación Física. Propuesta de innovación y gamificación basada en el videojuego $\begin{array}{lllllll}\text { Fortnite. } & \text { Sportis } & S c i & J & 5 & \text { (2), } & 323-350 .\end{array}$ https://doi.org/10.17979/sportis.2019.5.2.5257

Brown, A., y Smolenaers, E. (2018). Parents' interpretations of screen time recommendations for children younger than 2 years. Journal of Family Issues, 39(2), 406-429. DOI: https://doi.org/10.1177/0192513X16646595

Castro-Sánchez, M., Linares-Manrique, M., Sanromán-Mata, S., y Pérez Cortés, A. (2017). Análisis de los comportamientos sedentarios, práctica de actividad física y uso de videojuegos en adolescentes. Sportis. Scientific Journal of School Sport, Physical Education and Psychomotricity, 3(2), 241-255. DOI: https://doi.org/10.17979/sportis.2017.3.2.1746

Cole, T. J., Flegal, K. M., Nicholls, D., y Jackson, A. A. (2007). Body mass index cut offs to define thinness in children and adolescents: International survey. British Medical Journal $\begin{array}{lllll}\text { (Clinical } & \text { Research } & \text { Ed.), }\end{array}$ https://doi.org/10.1136/bmj.39238.399444.55 
Artículo Original. ¿Los menores españoles, en su tiempo libre, prefieren dispositivos electrónicos o actividad física? Vol. 6, n. ${ }^{\circ}$ 2; p. 347364, mayo 2020. A Coruña. España ISSN 2386-8333

Duggan, M. P., Taveras, E. M., Gerber, M. W., Horan, C. M., y Oreskovic, N. M. (2019). Presence of small screens in bedrooms is associated with shorter sleep duration and later bedtimes in children with obesity. Academic Pediatrics, 19(5), 515-519. DOI: https://doi.org/10.1016/j.acap.2018.11.004

Emond, J. A., Tantum, L. K., Gilbert-Diamond, D., Kim S. J., Lansigan, R. K. y BenjaminNeelon, S. E. (2018). Household chaos and screen media use among preschool-aged children: A cross-sectional study. BMC Public Health, 18, 1210. DOI: https://doi.org/10.1186/s12889-018-6113-2

Goodman, R. (2001). Psychometric properties of the strengths and difficulties questionnaire. Journal of the American Academy of Child \& Adolescent Psychiatry, 40(11), 1337-1345. DOI: https://doi.org/10.1097/00004583-200111000-00015

Hale, L., y Guan, S. (2015). Screen time and sleep among school-aged children and adolescents: A systematic literature review. Sleep Medicine Reviews, 21, 50-58. DOI: https://doi.org/10.1016/j.smrv.2014.07.007

Keane, E., Kelly, C., Molcho, M., y Gabhainn, S. N. (2017). Physical activity, screen time and the risk of subjective health complaints in school-aged children. Preventive Medicine, 96, 21-27. DOI: https://doi.org/10.1016/j.ypmed.2016.12.011

Lissak, G. (2018). Adverse physiological and psychological effects of screen time on children and adolescents: Literature review and case study. Environmental Research, 164, 149157. DOI: https://doi.org/10.1016/j.envres.2018.01.015

Livingstone, S., y Franklin, K. (2018). Families with young children and 'screen time'. Journal of Health Visiting, 6(9), 434-439. DOI: https://doi.org/10.12968/johv.2018.6.9.434

Maher, C., Virgara, R., Okely, T., Stanley, R., Watson, M., y Lewis, L. (2019). Physical activity and screen time in out of school hours care: An observational study. BMC Pediatrics, 19(1), 1-10. DOI: https://doi.org/10.1186/s12887-019-1653-x

Monsalve-Robayo, A., Mora-Caro, L. F., Ramírez-López, L. C., Rozo-Hernández, V., y Rojas-Puerto, D. M. (2017). Estrategias de intervención dirigidas a niños con trastorno negativista desafiante, una revisión de la literatura. Revista Ciencias De La Salud, 15(1), 105-127. DOI: https://doi.org/10.12804/revistas.urosario.edu.co/revsalud/a.5384

Para citar este artículo utilice la siguiente referencia: Boente-Antela, B.; Leirós-Rodríguez, R.; García-Soidán, J.L. (2020). ¿Los menores españoles, en su tiempo libre, prefieren dispositivos electrónicos o actividad física?. Sportis Sci J, 6 (2), 347-364. DOI: https://doi.org/10.17979/sportis.2020.6.2.6160

http://revistas.udc.es/ 
Nightingale, C. M., Rudnicka, A. R., Donin, A. S., Sattar, N., Cook, D. G., Whincup, P. H., y Owen, C. G. (2017). Screen time is associated with adiposity and insulin resistance in children. Archives of Disease in Childhood, 102(7), 612-616. DOI: https://doi.org/10.1136/archdischild-2016-312016

Oliver, M., Schofield, G. M., y Kolt, G. S. (2007). Physical activity in preschoolers. Sports Medicine, 37(12), 1045-1070. DOI: https://doi.org/10.2165/00007256-200737120-00004

Portolés-Ariño, A., y González-Hernández J. (2016). Perfiles adolescentes según orientación de metas: relación con conductas sedentarias. Sportis. Scientific Journal of School Sport, Physical Education and Psychomotricity, 2(2), 222-238. DOI: https://doi.org/10.17979/sportis.2016.2.2.1436

Ruiz, R. D., y Castañeda, M. A. (2016). Relación entre uso de las nuevas tecnologías y sobrepeso infantil, como problema de salud pública. RqR Enfermería Comunitaria, 4(1), 46-51.

Sánchez-Zafra, M.; Ramirez-Granizo, I.A.; Baez-Mirón, F.; Moreno-Arrebola, R.; FernándezRevelles, A.B. (2019). Análisis de la relación existente entre el uso de videojuegos y la práctica de actividad física. Sportis Sci J, 5 (1), 118-132. DOI: https://doi.org/10.17979/sportis.2019.5.1.3433

Sezen, H., Kandemir, H., Savik, E., BasmacÄ \pm Kandemir, S., Kilicaslan, F., Bilinc, H., y Aksoy, N. (2016). Increased oxidative stress in children with attention deficit hyperactivity disorder. Redox Report, 21(6), 248-253. DOI: https://doi.org/10.1080/13510002.2015.1116729

Tandon, P. S., Sasser, T., González, E. S., Whitlock, K. B., Christakis, D. A., y Stein, M. A. (2019). Physical activity, screen time, and sleep in children with ADHD. Journal of Physical Activity and Health, 16(6), 416-422. DOI: https://doi.org/10.1123/jpah.20180215

Twenge, J. M., Hisler, G. C., y Krizan, Z. (2019). Associations between screen time and sleep duration are primarily driven by portable electronic devices: Evidence from a populationbased study of US children ages 0-17. Sleep Medicine, 56, 211-218. DOI: https://doi.org/10.1016/j.sleep.2018.11.009

Para citar este artículo utilice la siguiente referencia: Boente-Antela, B.; Leirós-Rodríguez, R.; García-Soidán, J.L. (2020). ¿Los menores españoles, en su tiempo libre, prefieren dispositivos electrónicos o actividad física?. Sportis Sci J, 6 (2), 347-364. DOI: https://doi.org/10.17979/sportis.2020.6.2.6160 
Artículo Original. ¿Los menores españoles, en su tiempo libre, prefieren dispositivos electrónicos o actividad física? Vol. 6, n. ${ }^{\circ}$ 2; p. 347364, mayo 2020. A Coruña. España ISSN 2386-8333

World Health Organization. (2019). Global action plan on physical activity 2018-2030: More active people for a healthier world, Geneva, Switzerland: World Health Organization. 